night shifts in the current work schedule and the total duration of night shift work.

Results Chronic back pain, hypertension and thyroid disease were the most common diseases in the total population of nurses and midwives. The prevalence of thyroid disease was higher than in the general population of Polish women $(21.2 \%$ vs. $10 \%)$ and the relative risk of this disease increased with increasing duration of night shifts work. The analysis did not show significantly higher frequency of any disease or symptom in the night shifts nurses compared to the day workers. The analysis showed significantly increased risk of feet swelling in women working 8 or more night shifts per month $(\mathrm{OR}=8.55 ; 95 \%$ CI: $1.02-71.80)$.

Conclusions We did not find significant increased risk of any of the diseases or symptoms among night shifts nurses than among the women working only during the day. The increased prevalence of thyroid disease among the women with long duration of night shift work warrants further epidemiological studies.

\section{INCIDENCE OF SHARP-POINTED INJURIES IN PERSONNEL OF A HIGH SPECIALTY HOSPITAL FROM THE MEXICAN INSTITUTE OF SOCIAL SECURITY, 2004-2010}

C A J P Juarez-Perez, G Rodrigo Nieto. Instituto Mexicano Del Seguro Social, Distrito Federal, Mexico Distrito Federal, Mexico

\subsection{6/oemed-2013-101717.43}

Objective To determine the incidence of sharp pointed injuries by working category and its characteristics.

Material and Methods Information of sharp pointed injuries from 2004 to 2010 was collected using EPINet, which registered: working traits, service, description of the accident, object, and anatomical part among others.

Results We recorded 181 injuries, with a mean (SD) [min-max] of: age 36 (8.5) [18-56] years; length of service in the hospital 10.6 (7.7) [0.5-27] years. The greatest proportions of injuries were in the departments of: Adolescents $31.5 \%$, NICU $25 \%$ and Operating Room 16\%. The first working shift had the largest amount of accidents with $71.7 \%$. Moderate lesions were the most frequent with $64 \%$, followed by superficial $21 \%$ and severe $15 \%$. Of all the personnel, 55\% didn't use gloves, $41 \%$ used one pair and $5 \%$ used double pair of gloves; $49 \%$ was injured in the right hand and 48.6 in the left hand. The main working categories injured were: nurses $48 \%$, medical residents $23.2 \%$, general services $16.6 \%$, and physicians $6.6 \%$. The incidences were: medical residents $31.5 \%$, nurses $13.8 \%$, general services $11.5 \%$ and physicians $7 \%$. Taking into account the ones with lower risk, we observed a greater ratio of incidence in the medical residents: 4.5 vs physicians and 2.3 vs nurses.

Conclusions Medical residents are in greater risk of having sharp pointed injuries followed by nurses and general services. It is necessary to identify the causes and take preventive measures as well as developing permanent epidemiological surveillance of these risks.

\section{BURNOUT SYNDROME IN MEDICAL RESIDENTS OF A SCHOOL HOSPITAL AND THE ORGANISATIONAL ERGONOMY FACTORS}

C A F Fabichak, Silva-Junior, Morrone. Faculdade de Ciências Médicas da Santa Casa de São Paulo, São Paulo, Brazil

10.1136/oemed-2013-101717.44
Objectives To describe the presence of Burnout syndrome among doctors in residency medical postgraduate and to evaluate in organisational ergonomics factors in the framework of a school hospital which predict that syndrome.

Methods Cross-sectional study performed in a school hospital in São Paulo, southeastern of Brazil. Participants were 24 doctors in two-years medical residency program of Internal Medicine. They answered questionnaire about sociodemographic data, clinical complaints, occupational informations and perception of work organisational predictors for Burnout Syndrome. The outcome was analysed by their response of Maslach Burnout Inventory (MBI).

Results Most of the participants were female (58.3\%), single $(91.7 \%)$, living alone $(66.7 \%)$, being the first year of the program (54.2\%), working 60 hours/week (83.3\%), have another job $(62,5 \%)$ and reported high number of complaints (54.2\%). The mean age of participants was 26 years old. The Burnout Syndrome was characterised in 50\% of participants. The frequence of perceived work organisation stressors were: excessive demands (66.7\%) with high control at work (79.2\%), low recognition by preceptors $(87.5 \%)$, low valuation by the institution $(79.2 \%)$ and low support from colleagues (62.5\%). Most of them considered their work important $(70.8 \%)$.

Conclusions These workers presented Burnout syndrome as reported in other studies with similar population. The perception of predictors for this condition in their postgraduate program shows a stressful situation. More than employees, they have a study bond with this program and negative impacts in their learning could happen. It is necessary to analyse organisational factors to create policies aimed to minimise occupational and psychosocial stressors.

\section{ESTIMATION OF THE NATIONAL INCIDENCE OF NEEDLESTICK INJURY IN TAIWAN HEALTHCARE WORKERS}

${ }^{1} \mathrm{H} \mathrm{C} \mathrm{Wu},{ }^{2}$ Shiao, ${ }^{3} \mathrm{Guo},{ }^{4} \mathrm{Chin} .{ }^{1} \mathrm{H}$ sin Sheng College of Medical Care and Management, Longtan Township, Taoyuan County, Taiwan; ${ }^{2}$ Department of Nursing, Colledge of Medicine, National Taiwan University., Taipei, Taiwan; ${ }^{3}$ Department of Environmental and Occupational Medicine, NTU College of Medicine, National Taiwan University, Taipei, Taiwan; ${ }^{4}$ Institute of Occupational Medicine and Industry Hygiene, National Taiwan University, Taipei, Taiwan

\subsection{6/oemed-2013-101717.45}

Objectives The purpose of this study was to determine which of the following denominators, number of health care workers, number of hospital beds, and number of inpatient-days would predict best of the national number of needlestick injury (NSIs) and the national number of biological exposures to specific bloodborne pathogens.

Methods Four years after our first attempt at calculating the national incidence of NSIs in Taiwan healthcare workers (HCWs) from 14 hospitals, using Exposure Prevention Information Network (EPINet) data, we are now able to analyse the much larger dataset reported from 49 hospitals that consist of 44,939 HCWs.

Results A total of 1,837 NSIs were reported between Jan 1st and Dec 31th 2011. The annual number was estimated to be 5,663 NSIs per hospital size, 8,319 per HCWs, and 7,828 per inpatientday; indicating similar estimates using different denominators. The estimated annual frequency of pathogen-specific NSIs was 970 for hepatitis B, 1,094 for hepatitis C, and 99 for HIV. 